\title{
LA POLIITICA EXTERIOR DE ESTADOS UNIDOS HACIA BRASIL: CAMBIOS Y CONTINUIDADES EN LA ADMINISTRACIÓN BIDEN
}

\author{
THE FOREIGN POLICY OF THE UNITED STATES \\ TOWARDS BRAZIL: \\ CHANGES AND CONTINUITIES IN THE BIDEN \\ ADMINISTRATION
}

\author{
Daniel Morales Ruvalcaba ${ }^{1}$ \\ ORCID: 0000-0002-4304-3831
}

\section{RESUMEN}

Durante sus dos primeros años como presidente de Brasil, Jair Bolsonaro dio la máxima prioridad al ligamen con Estados Unidos, de modo que logró un entendimiento extraordinario con el mandatario Donald Trump. Sin embargo, la llegada de Joseph Biden a la presidencia ha tenido un significativo impacto en dicho entendimiento. Aquí se demuestra que las relaciones brasileño-estadounidenses con Trump y Bolsonaro se enmarcaron en sus programas de gobierno populistas y que fueron impulsadas por su mutua comprensión personal, hecho que alteró las prioridades (pero no el contenido) de la agenda bilateral. El arribo de Biden y su distanciamiento ideológico de Bolsonaro no implican necesariamente un enfriamiento en la relación bilateral, sino un redireccionamiento a los canales institucionales gracias a los mecanismos preexistentes entre Estados Unidos y Brasil, así como un retorno a las prioridades históricas: primero los asuntos económicos, luego los energéticos y medioambientales, posteriormente los militares y, por último, los sociales. En esta investigación, se ha implementado la metodología deductiva en el estudio de dos dimensiones: una discursiva, al examinar los discursos oficiales más importantes de Trump, Bolsonaro y Biden en las relaciones brasileñoestadounidenses, con el objetivo de identificar los cambios en sus percepciones y elementos primordiales; y otra histórica, al estudiar el desempeño de las instituciones bilaterales en las áreas antes referidas, con la finalidad de detectar los patrones de continuidad que van más allá de las administraciones presidenciales.

Palabras clave: Brasil; Donald Trump; Estados Unidos; Jair Bolsonaro; Joseph Biden; Política exterior

\section{ABSTRACT \\ During his first two years as president of Brazil, Jair Bolsonaro gave the relationship with the United States the highest priority, achieving an extraordinary understanding with the head of state Donald Trump. However, the arrival of Joseph Biden to the presidency has had a significant impact on that understanding. Here it is shown that the Brazilian-American relations with Trump and Bolsonaro were}

1 Centro de Estudios Latinoamericanos, Universidad Sun Yat-sen 中山大学 (China). Investigador. Miembro del Sistema Nacional de Investigadores (México). Doctor en Ciencias Sociales. Correo electrónico: morales@mail.sysu.edu.cn 
framed in their populist government programs and they were driven by their mutual personal agreement, altering the priorities (but not the content) of the bilateral agenda. The entrance of Biden and his ideological distancing from Bolsonaro does not necessarily imply a cooling in the bilateral relationship, but rather a redirection to institutional channels thanks to the pre-existing mechanisms between the United States and Brazil, as well as a return to historical priorities: economic affairs first, then energy and environmental issues, later military cooperation, and finally social topics. In this research, the deductive methodology has been implemented in the study of two dimensions: a discursive one, by examining the most important official speeches of Trump, Bolsonaro and Biden in Brazilian-American relations, with the aim of identifying changes in their perceptions and priorities; and another historical one, when studying the performance of bilateral institutions in the aforementioned areas, in order to detect continuity patterns that go beyond presidential administrations.

Keywords: Brazil; Donald Trump; Foreign policy; Jair Bolsonaro; Joseph Biden; United States

\section{Introducción ${ }^{2}$}

Las relaciones entre Brasil y Estados Unidos son muy complejas y diversas, además de considerarse unas de las más antiguas y amistosas del continente americano, pues EE. UU. fue el primer país en reconocer la independencia de Brasil (en 1824) y uno de los primeros en abrir una representación diplomática en territorio brasileño (en 1825). Los históricos vínculos de armonía y entendimiento han llevado a hablar de una "alianza no escrita" entre ambas naciones (Burns, 1966).

Dicho entendimiento cuenta con importantes bases económicas y sociales. Estados Unidos fue uno de los principales socios comerciales de Brasil en los siglos XIX y XX, lo cual motivó a que, en 1878, los dos países firmaran un Agreement Concerning Trade-Marks para la protección recíproca de marcas de manufactura y comercio; en 1909, celebraron una convención de arbitraje para la solución de diferencias; en 1914, signaron el Treaty for the Advancement of General Peace para promover el progreso y la amistad entre ambas naciones; en 1976, crearon el Brazil-U.S. Business Council (BUSBC) para fomentar las asociaciones comerciales entre la sociedad civil. En otros términos, esta relación bilateral ha sido construida a lo largo de los siglos y, gradualmente, ha incorporado nuevos y diversos actores. Muestra de la densidad en dichos nexos es, por ejemplo, que hoy Estados Unidos alberga la mayor cantidad de consulados brasileños en el exterior (10 en total).

Efectivamente, las últimas décadas se caracterizan por un diálogo político muy intenso, pues, desde el gobierno de Fernando Henrique Cardoso (1995-2002),

2 Este trabajo ha contado con la colaboración de Xiaomin Xu, quien ha apoyado en la búsqueda de artículos especializados y notas periodísticas; recopilación informativa; sistematización de datos y creación de gráficas. A ella mi reconocimiento y gratitud por su valiosa contribución. 
todos los presidentes brasileños han viajado a Estados Unidos en al menos una ocasión; en el caso de los mandatarios estadounidenses, desde Jimmy Carter (1977-1981), todos ellos han realizado visitas oficiales a Brasil, con la notaria excepción de Donald Trump (no obstante, su vicepresidente Mike Pence llevó a cabo una visita oficial el 27-28 de junio de 2018 y el secretario de Estado Mike Pompeo hizo dos viajes, uno el 1-2 de enero de 2019 y otro el 18 de septiembre de 2020).

Mientras Donald Trump se encontraba al frente del Gobierno de Estados Unidos, Jair Bolsonaro fue electo como presidente de Brasil. Durante sus dos primeros años, Bolsonaro dio la mayor prioridad al ligamen con Estados Unidos y logró un extraordinario entendimiento con Trump. Sin embargo, la llegada de Joe Biden a la presidencia ha tenido un impacto directo en dicho entendimiento. En ese sentido, es importante evaluar, ¿en qué estado se encuentran las relaciones entre Brasil y Estados Unidos al momento de la transición Trump-Biden? ¿Cuáles son las pautas para el reordenamiento de la agenda de trabajo entre los Gobiernos de Brasil y Estados Unidos, en términos económicos, de energía/ cambio climático, defensa y asuntos sociales?

Aquí se demuestra que las conexiones brasileño-estadounidenses con los presidentes Trump y Bolsonaro se enmarcaron en sus programas de gobierno populistas y que fueron impulsadas por su mutuo entendimiento personal, alterando las prioridades de la agenda bilateral, pero no su contenido. La llegada de Biden y su distanciamiento ideológico con Bolsonaro no implica necesariamente un enfriamiento en la relación, sino un redireccionamiento a los canales institucionales, gracias a los mecanismos preexistentes entre Estados Unidos y Brasil, así como un retorno a las prioridades históricas.

Para demostrarlo, este trabajo ha sido organizado en cuatro partes: en la primera, se exponen las asimetrías de poder nacional históricamente existentes entre Estados Unidos y Brasil, las cuales sirven para enmarcar el pensamiento de Jair Bolsonaro y su anhelo por profundizar la relación bilateral; en la segunda, se da cuenta del amplio entendimiento desarrollado entre los presidentes Bolsonaro y Trump, así como de la alteración de las prioridades en la agenda bilateral, todo ello sin salir de sus programas populistas de gobierno; en la tercera, se analiza el rol y las percepciones de Joseph Biden en las relaciones brasileñoestadounidenses durante sus años como vicepresidente; $y$, en la última parte, se examinan los cambios y continuidades en las relaciones bilaterales con la administración Biden, a partir de la revisión del estado de las instituciones bilaterales existentes en cuatro áreas temáticas: economía, energía/cambio climático, defensa y asuntos sociales. 
En esta investigación, se ha implementado una metodología deductiva en el estudio de dos dimensiones, una discursiva y otra histórica, para inferir las características de la política exterior de la administración Biden con Brasil. Primeramente, se ha aplicado la metodología deductiva a la dimensión discursiva, al examinar los discursos oficiales más importantes de Trump, Bolsonaro y Biden sobre las relaciones brasileño-estadounidenses, con el objetivo de identificar sus percepciones y prioridades. Para mostrar con mayor claridad los resultados del análisis de estos discursos, se ha introducido aquí la técnica de "nube de palabras". Posteriormente, se ha empleado la metodología deductiva en la dimensión histórica, al estudiar, de manera puntual, el desempeño de las instituciones bilaterales que han conducido los vínculos económicos, energético/medioambientales, militares y sociales entre Brasil y Estados Unidos en las últimas décadas, con el fin de observar los patrones de continuidad que trascienden las administraciones presidenciales. En términos temporales, el estudio concluye el 27 de enero de 2021, una vez que Biden concluye su primera semana como presidente de Estados Unidos y cumplimenta la firma de decenas de órdenes ejecutivas con las que traza el rumbo de su gobierno.

\section{Asimetría en las relaciones Estados Unidos- Brasil: entre la autonomía y el alineamiento}

Las relaciones brasileño-estadounidense se caracterizan por una marcada asimetría en favor de Estados Unidos y en detrimento de Brasil. Como se muestra en el gráfico 1, el World Power Index ${ }^{3}$ ayuda a mostrar las significativas e históricas diferencias entre el cúmulo de poder nacional de Estados Unidos, que nunca ha descendido de 0.950 , y de Brasil, cuyos niveles más altos se registraron en el lapso 2011-2014, con valores ligeramente superiores 0.765 , en un momento en el que el país sudamericano era visto como una potencia emergente (Gratius, 2008; Cooper \& Antkiewicz, 2008; Scholvin, 2010; Schweller, 2011; Hurrell, 2012; Fonseca, Paes, \& Cunha, 2016; Zakol, 2019).

3 El World Power Index (WPI) se define como la expresión numérica, la cual calcula la acumulación de capacidades nacionales que posee un Estado para ejercer su poder en el sistema internacional. Está compuesto por tres subíndices - Índice de Capacidades Materiales, Índice de Capacidades Semimateriales e Índice de Capacidades Inmateriales - que miden diferentes dimensiones del poder nacional (Rocha \& Morales, 2018). Si bien, en términos históricos, resultan relevantes los datos previos a 1975, la base de datos del WPI solo posee información a partir de dicho año. 
Gráfico 1.

World Power Index para Estados Unidos y Brasil, 1975-2020

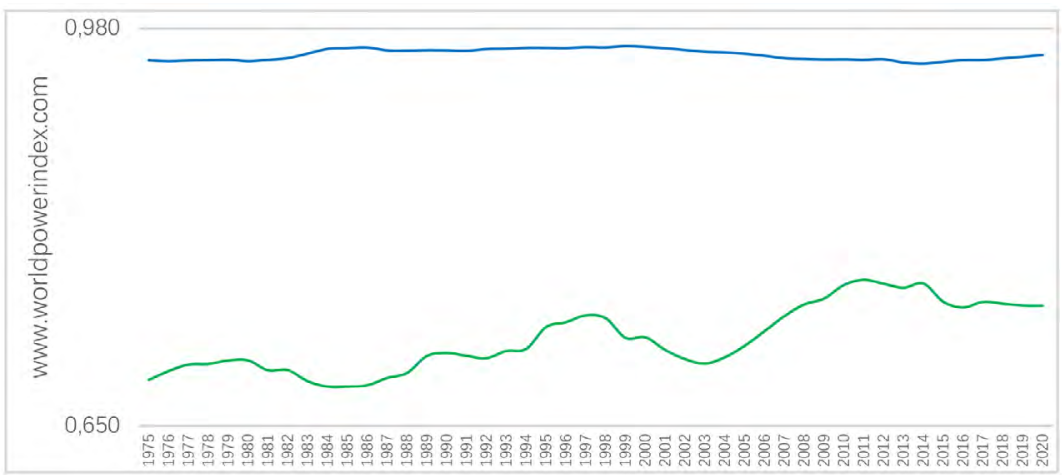

Elaboración propia: Daniel Morales Ruvalcaba, con la colaboración de Xiaomin Xu.

Fuente: www.worldpowerindex.com

A pesar de que su ascenso se detuvo en la segunda mitad de la década de los 10, Brasil es el país con la más grande dotación de poder de América Latina, pues posee las mayores cifras en territorio, población y economía de la región, por mencionar los rubros más relevantes. Esto significa que, por un lado, tiene el cúmulo de poder suficiente para protagonizar en el nivel global y, por otro, es un actor líder en el nivel regional (Nolte, 2006; Soares de Lima \& Hirst, 2006; Saraiva, 2012; Morales, 2020). En ese sentido, Brasil no solo sigue siendo un socio estratégico en términos geopolíticos y geoeconómicos para otras naciones que buscan vincularse con América Latina, sino que es una potencia latinoamericana, la cual, tradicionalmente, se ha destacado por desempeñar una política exterior altiva, buscar la ampliación de su presencia en el mundo y procurar el equilibrio en sus relaciones internacionales.

Debido a la asimetría de poder con Estados Unidos y al propio predominio de Brasil en elámbito regional, una de las formas de entender las relaciones brasileñoestadounidenses ha sido a través de la histórica oscilación entre los extremos de autonomía y alineamiento (Soreanu, 2008). Con la llegada de Jair Bolsonaro a la presidencia en enero de 2019, se ha implementado una nueva política exterior (Frenkel, 2018; Caballero \& Crescentino, 2020; Ribeiro Hoffmann, 2020), que tiene como principales artífices al ministro de Relaciones Exteriores, Ernesto Araújo, y a los hijos del mandatario, Flávio y Eduardo Bolsonaro, todos ellos directamente influidos por el polémico intelectual de ultraderecha Olavo de Carvalho. Uno de los principales rasgos de la política exterior bolsonarista es su extremo alineamiento a Estados Unidos, caracterizado como americanismo ideológico (Saraiva \& Silva, 2019; Mongan, 2020). 


\section{Relación Bolsonaro-Trump: entre el populis- mo y voluntarismo presidencial}

Durante sus dos primeros años como presidente de Brasil, Jair Bolsonaro dio la mayor prioridad a la relación con Estados Unidos y, especialmente, con Donald Trump: de los 13 viajes internacionales que el primero ha hecho entre enero de 2019 y enero de 2021, cuatro han tenido por destino Estados Unidos. En todas estas visitas, Bolsonaro tuvo reuniones con Trump, excepto en su viaje a Dallas, 14-16 de mayo de 2019, cuando fue premiado como la persona del año por la Brazilian-American Chamber of Commerce. Además de sus idas a Norteamérica, Bolsonaro celebró un encuentro más con el mandatario estadounidense, durante su participación en la cumbre del G20 en Osaka (cuadro 1). Con todo esto, el brasileño consiguió convertirse en uno de los líderes latinoamericanos que más veces se entrevistó con Trump.

Cuadro 1.

Reuniones entre el presidente estadounidense Donald Trump y el presidente brasileño Jair Bolsonaro, 2019-2020

\begin{tabular}{|l|l|l|}
\hline Fechas & Ciudad & Documentos relevantes \\
\hline $\begin{array}{l}\text { 18-20 de marzo } \\
\text { de } 2019\end{array}$ & $\begin{array}{l}\text { Washington, } \\
\text { D. C., Estados } \\
\text { Unidos }\end{array}$ & $\begin{array}{l}\text { Declaraciones del presidente Trump y } \\
\text { el presidente Bolsonaro de la República } \\
\text { Federativa de Brasil en conferencia de } \\
\text { prensa conjunta: http://bit.ly/368Yswp. } \\
\text { Declaración conjunta del presidente Donald } \\
\text { J. Trump y el presidente Jair Bolsonaro: } \\
\text { http://bit.ly/3c7JGKr. }\end{array}$ \\
\hline $\begin{array}{l}\text { 27-29 de junio de } \\
2019\end{array}$ & Osaka, Japón & $\begin{array}{l}\text { Declaraciones del presidente Trump y } \\
\text { el presidente Bolsonaro de la República } \\
\text { Federativa de Brasil antes de la reunión } \\
\text { bilateral: http://bit.ly/369pG68. }\end{array}$ \\
\hline $\begin{array}{l}\text { 23-24 de } \\
\text { septiembre de } \\
2019\end{array}$ & $\begin{array}{l}\text { Eueva York, } \\
\text { Estados } \\
\text { Unidos } \\
\text { una reunión multilateral sobre la República } \\
\text { Bolivariana de Venezuela: http://bit. } \\
\text { ly/3psiTfL. }\end{array}$ \\
\hline $\begin{array}{l}\text { 7-10 de marzo de } \\
2020\end{array}$ & $\begin{array}{l}\text { Miami, } \\
\text { Estados } \\
\text { Unidos }\end{array}$ & $\begin{array}{l}\text { Declaración conjunta del presidente Donald } \\
\text { Trump y el presidente Jair Bolsonaro: } \\
\text { http://bit.ly/2KK2CDz. }\end{array}$ \\
\hline
\end{tabular}

Elaboración propia: Daniel Morales Ruvalcaba, con la colaboración de Xiaomin Xu. 
El encuentro bilateral más importante entre los presidentes Trump y Bolsonaro tuvo lugar en Washington, D. C., el 18 y 19 de marzo de 2019, pues los discursos pronunciados por ellos tuvieron amplia cobertura y difusión en los medios comunicativos de ambos países.

El discurso de Donald Trump fue de 988 palabras. Si se excluyen los vocablos que han sido mencionados solo una ocasión, así como los recursos protocolarios para eventos de esta naturaleza (como President, Brazil, United States, Bolsonaro), los más nombrados fueron: people (9), country (6), great (6), incredible (6), working (6), nations (5), together (5). Como puede observarse, todos estos son calificativos sobre el ligamen brasileño-estadounidense, pero no sugieren una temática concreta en la agenda de trabajo con Brasil. El primer tema que se puede encontrar y se convierte en eje de su discurso es Venezuela (aludido tres veces), lo cual habla de la instrumentalización de Brasil en la campaña trumpista contra la nación sudamericana. A partir de dicha colaboración, Trump expresó: "Como le dije al presidente Bolsonaro, también tengo la intención de designar a Brasil como un Major non-NATO Ally, o incluso posiblemente, si se empieza a pensar en ello, tal vez un aliado de la OTAN" (The White House, 2019a). Así, a lo largo del alegato, se puede corroborar que la cooperación militar quedó posicionada en el primer lugar (que, además del asunto del Venezuela, contempla áreas como terrorismo, crimen trasnacional, tráfico de armas y humano), mientras que otros temas de relevancia histórica para los empresarios y demás actores de la sociedad fueron apenas referidos por Trump.

Por su parte, el discurso de Bolsonaro fue de 737 palabras (versión en inglés). En este caso, al excluir los términos que han sido mencionados en una ocasión y los elementos protocolarios (tales como Brazil, President, United States, America, US), los más citados resultaron carentes de contenido — today (5), part (4), side (4), two (4), will (4) -; de tal forma, las primeras referencias a una agenda de trabajo conjunta se encontraron en business (3), cooperation (3), forum (3). A pesar de la ausencia de un eje argumentativo, con su exposición, Bolsonaro situó el enlace con Estados Unidos en un altísimo nivel, al declarar: "Este encuentro nuestro hoy restaura una vieja tradición de asociación. Y, al mismo tiempo, comienza un nuevo capítulo de cooperación entre Brasil y los Estados Unidos. [...] Es hora de superar la vieja resistencia y explorar el mejor potencial que hay entre Brasil y Estados Unidos" (The White House, 2019a), pero todo esto sustentado no en una estrategia amplia, sino en el hecho de que "Siempre he admirado los Estados Unidos de América. Y este sentimiento de admiración acaba de aumentar después de que usted [Trump] asumió el cargo, en la presidencia. [...] Después de todo, es justo decir que, hoy en día, Brasil tiene un presidente que no es antiestadounidense, lo cual es realmente sin precedentes en las últimas décadas" (The White House, 2019a). 
Para dimensionar el contenido del diálogo entre los mandatarios estadounidense y brasileño, se presentan, en el gráfico 2, un par de nubes de palabras, las cuales son una muestra del discurso de dos líderes populistas quienes, en materia de política exterior, evidencian retórica en su agenda de trabajo, una formulación de dicho tipo de política más centralizado, justifican sus decisiones en la voluntad general y extrapolan las preferencias populares a la acción internacional (Chryssogelos, 2017; Destradi \& Plagemann, 2019; Wehner \& Thies, 2020). Así, durante el 2019 y 2020, las relaciones brasileño-estadounidenses fueron esencialmente conducidas por el voluntarismo de sus líderes $\mathrm{y}$, de manera más específica, por el americanismo ideológico de Bolsonaro.

Gráfico 2.

Nube de palabras comparada.

Discursos de los presidentes Trump (izquierda) y Bolsonaro (derecha) en la reunión bilateral del 19 de marzo de 2019 en Washington, D. C.
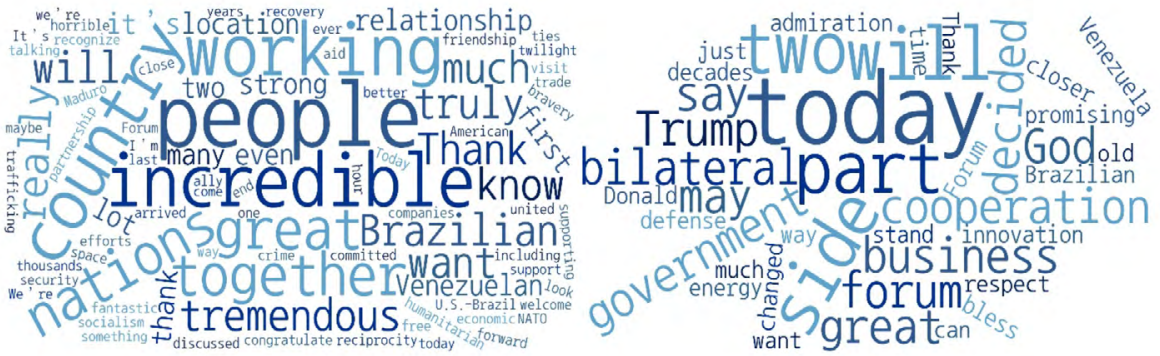

Elaboración propia: Daniel Morales Ruvalcaba, con la colaboración de Xiaomin Xu.

Fuente: The White House, 2019a.

Es cierto que, en las últimas tres décadas, 11 naciones latinoamericanas han firmado acuerdos de libre comercio con Estados Unidos ${ }^{4}$, mientras que Brasil ha mantenido estancadas sus negociaciones comerciales, desaprovechando la oportunidad de avanzar hacia una mayor liberalización (Hakim, 2019; Villarreal, Meyes \& Schwarzenberg, 2020). Sin embargo, a pesar de los múltiples encuentros entre Trump y Bolsonaro, estos no impidieron acciones negativas contra Brasil como el establecimiento de aranceles a su acero y aluminio o su retiro de la lista de países en desarrollo por parte de Estados Unidos.

4 Chile (2003), Colombia (2006), Costa Rica (2004), El Salvador (2004), Guatemala (2004), Honduras (2004), México (1992), Nicaragua (2004), Panamá (2007), Perú (2006) y República Dominicana (2004). 
De tal forma, la carencia de un encuadre de la relación brasileño-estadounidense en una estrategia diplomática más amplia por parte del gobierno Bolsonaro provocó profundos rechazos de políticos de alto nivel y antiguos ministros de Relaciones Exteriores de Brasil - como Fernando Henrique Cardoso, Aloysio Nunes Ferreira, Celso Amorim, Celso Lafer y José Serra, entre otros-, quienes señalaron la violación sistemática de los principios rectores de política exterior consagrados en la Constitución de Brasil. Aludiendo directamente al irrestricto alineamiento del gobierno Bolsonaro a Estados Unidos, ellos escribieron:

La independencia nacional no puede conciliarse con la subordinación a un gobierno extranjero cuyo programa político admitido es promover su interés por encima de todas las demás consideraciones. Aliena la independencia del gobierno que se declara aliado de ese país, asumiendo como propia una agenda que amenaza con arrastrar a Brasil a conflictos con naciones con las que mantenemos relaciones de amistad e interés mutuo (Cardoso et al., 2020).

Como señalan los expertos, el Itamaraty ha visto comprometidos su liderazgo y prestigio durante el gobierno Bolsonaro y, de seguir esa tendencia, Brasil terminaría marginado de la escena mundial, lo cual tampoco es del interés de Estados Unidos (Committee on Ways and Means, 2020). No obstante, la administración Trump llegó a su fin y, con ello, se ha dado en Washington un viraje en política internacional, el cual se ha traducido también en el reajuste de su relación con Brasilia.

\section{Relación de Joseph Biden con Brasil: percep- ciones y rol como vicepresidente}

Como se estableció, Brasil es la principal potencia latinoamericana y, por tanto, siempre ha tenido un peso significativo en las relaciones hemisféricas (Bodman, Wolfersohn \& Sweig, 2011); de ahí que Brasil haya ocupado una posición destacada no solo para Donald Trump, sino también para Barack Obama (20092017). De hecho, durante la administración Obama, Brasil fue — después de México - el país latinoamericano más visitado por los altos funcionarios responsables de la política exterior (ver gráfico 3): Obama viajó a Brasil del 19 al 21 de marzo de 2011 para reunirse con la presidenta Dilma Rousseff; los secretarios de Estado realizaron seis visitas al país, Hillary Clinton (2009-2013) en cuatro ocasiones ${ }^{5}$ y John Kerry (2013-2017) solo dos veces ${ }^{6}$; mientras que el vicepresidente Joe Biden se movilizó a dicho destino en tres ocasiones.

5 Clinton: 1) 2-3 de marzo de 2010, 2) 1 de enero de 2011, 3) 16-18 de abril de 2012, 4) 20-22 de junio de 2012.

6 Kerry: 1) 13 de agosto de 2013, 2) 5-6 de agosto de 2016. 
Gráfico 3.

Países latinoamericanos visitados por el presidente, la/el titular de la Secretaría de Estado y el vicepresidente de Estados Unidos durante la administración Obama, 2009-2017

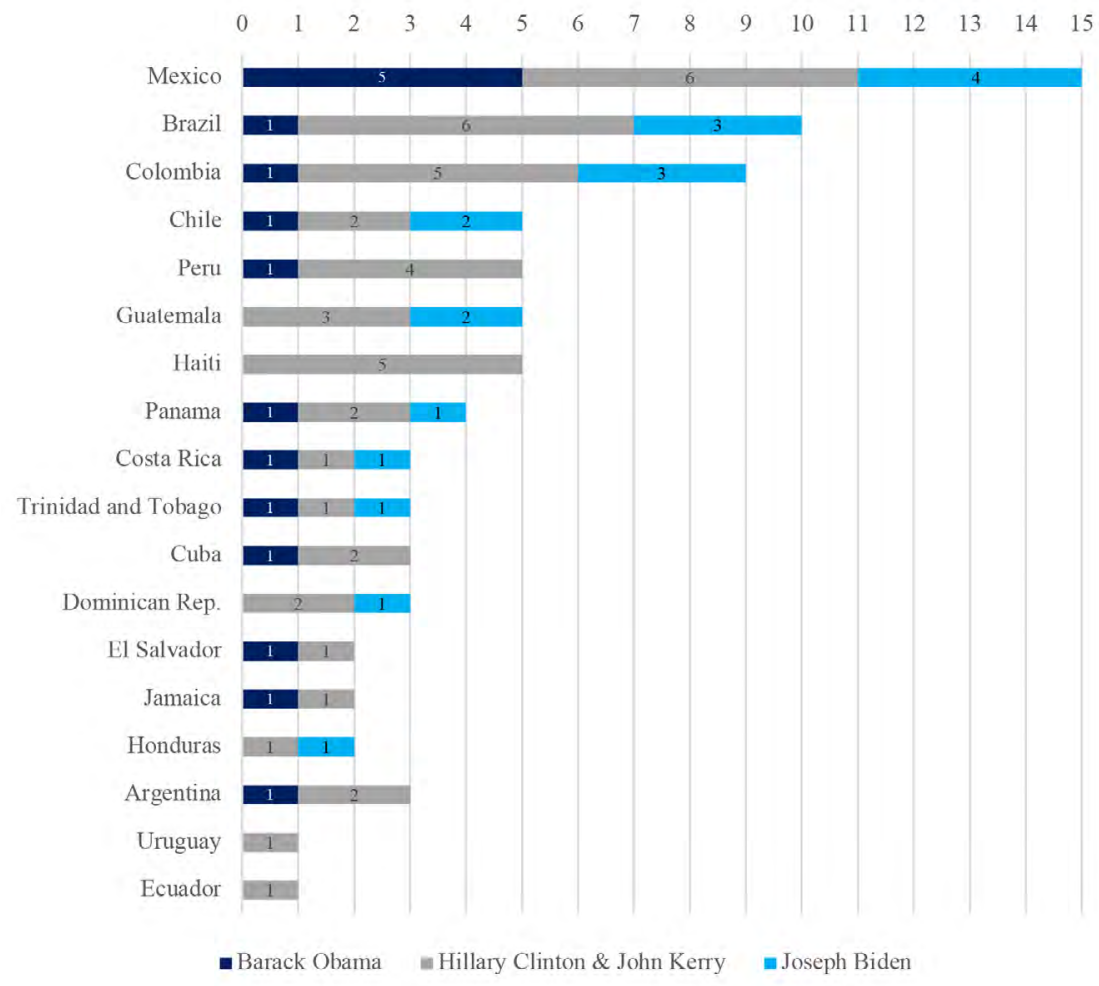

Elaboración propia: Daniel Morales Ruvalcaba, con la colaboración de Xiaomin Xu.

Es importante señalar que Biden es un entusiasta del panamericanismo (Padilla, 1954; Briceño, 2016, 2017; Correa, 2020) y, en esto, considera a Brasil como un actor valioso con el cual es imprescindible que Estados Unidos desarrolle relaciones armónicas y colaborativas, pues dijo: “Ambos podemos seguir prosperando, profundicemos o no nuestras relaciones económicas. Pero imagínense, imagínense lo que estas dos economías dinámicas podrían hacer con un mayor comercio e inversión para nuestra gente, para el hemisferio, para el mundo" (Biden, 2013). Por ello, cada una de sus tres visitas a Brasil estuvieron orientadas a impulsar la agenda bilateral y, especialmente, a generar marcos 
de entendimiento con Brasilia para la cooperación en asuntos hemisféricos y globales.

En cuanto a las actividades de Biden, en sus viajes a Brasil, estas se desarrollaron de la siguiente forma:

1. 29-31 de mayo de 2013: este fue un recorrido intenso que tuvo varios actos significativos como la visita a una favela de Río de Janeiro, el encuentro con líderes empresariales del sector energético y reuniones importantes con la presidenta Dilma Rousseff.

2. 16-18 de junio de 2014: en esta ocasión asistió a la Copa Mundial de la FIFA y nuevamente sostuvo reuniones con la presidenta Rousseff para dar continuidad a las relaciones entre ambos países.

3. 1-2 de enero de 2015: participó en la inauguración del segundo mandato de Rousseff y planteó la necesidad de seguir impulsando la cooperación en asuntos bilaterales, regionales y globales.

Entre dichas reuniones, la más importante fue la primera (2013), pues Biden pronunció un histórico discurso, el cual no solo estructuró la agenda bilateral durante la administración Obama, sino que sirve de guía para prefigurar el curso de la asociación brasileño-estadounidense en la era post-Trump.

La alocución del vicepresidente Biden fue de 3387 palabras. En este caso, al eliminar los vocablos que son mencionados una y dos veces, así como los recursos protocolarios (como Brazil, countries/country, President, United States), es posible encontrar un discurso con mayor contenido temático, el cual refleja un entendimiento mucho más amplio y complejo de la relación BrasilEstados Unidos (ver gráfico 4). Sobresalen aquí términos como world (25), economic + economies + economy $(7+4+4$, esto es 15 en total), democracy + democratic (5 +5, 10 en total), hemisphere (9), investment (9), Americas (8), energy (8), trade (7). 
Gráfico 4. Nube de palabras.

Palabras del vicepresidente sobre las relaciones entre Estados Unidos y Brasil, 2013

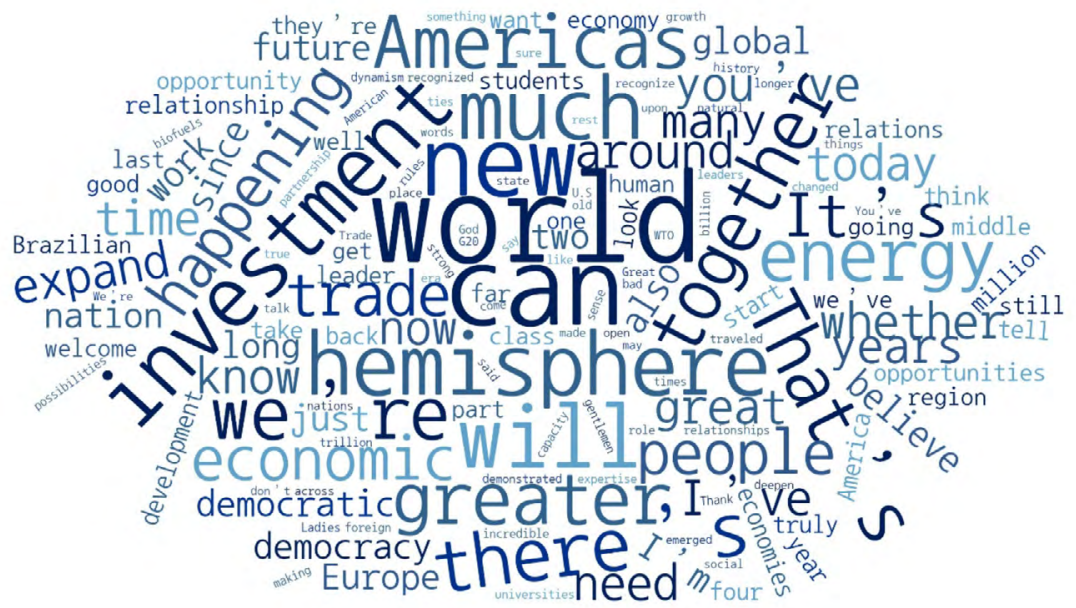

Elaboración propia: Daniel Morales Ruvalcaba, con la colaboración de Xiaomin Xu.

Fuente: Biden, 2013.

Es comprensible que la primera palabra en cantidad de repeticiones sea world, pues Biden (2013) declaró: "Brasil ya no puede hablar de ser una potencia emergente: ustedes han emergido y todos se han dado cuenta". En otros términos, para el nuevo presidente de Estados Unidos, Brasil es una potencia que amerita un reconocimiento especial y que resulta un actor fundamental en la política mundial. Ahora bien, cuatro áreas prioritarias en la agenda bilateral pueden ser identificadas en el discurso de Biden, tanto cuantitativa (por repetición de palabras) como cualitativamente (a partir de la estructura del discurso): 1) relación económica, con especial referencia al comercio y las inversiones; 2) cooperación energética, concretamente en lo que refiere a biocombustibles y energía renovable; 3 ) coordinación política para la gestión de asuntos regionales y globales; 4) profundización de los vínculos sociales, esto entendido a través de los intercambios científicos y educativos.

Cabe anotar que la relación brasileño-estadounidense se deterioró significativamente en el segundo semestre de 2013, debido a las revelaciones de Edward Snowden sobre el espionaje que los servicios de inteligencia de Estados Unidos realizaron en los correos electrónicos y teléfonos de la presidenta Dilma Rousseff. El vicepresidente Biden buscó zanjar este problema con sus visitas a Brasil en el 2014 y 2015, las cuales, si bien fueron de menor relevancia, 
sostuvieron siempre un diálogo directo con Rousseff. Por ello, el discurso de 2013 prevalece como el más importante para conocer las prioridades de Biden en la unión con Brasil y, a partir de los elementos analizados en este, es posible prefigurar la agenda del presidente con Brasil.

El paso siguiente es examinar los cambios y continuidades de las relaciones bilaterales, con base en la revisión del estado de las instituciones bilaterales. Cabe subrayar, en términos temporales, que el relevamiento de información del siguiente segmento concluye el 27 de enero de 2021, una vez que Biden finaliza su primera semana como presidente de Estados Unidos y traza el rumbo de su administración a través de la firma de decenas de órdenes ejecutivas.

\section{Las relaciones brasileño-estadounidenses en la administración Biden: análisis institucional}

Un aspecto que caracteriza al presidente Biden es su tradicional apego a las normas y las instituciones, lo cual marca una enorme diferencia con el mandatario anterior. Esto significa que mientras Trump — de orientación populista - condujo las relaciones con Brasil a partir de su buen entendimiento con Bolsonaro, Biden - quien sobresale por ser un liberal en política internacional-, previsiblemente, tomaría como base de acción las normas e instituciones creadas conjuntamente. Así, la cooperación con Brasil sería reencausada e impulsada desde los marcos bilaterales preexistentes.

Ahora bien, debido a la importancia que adquieren las instituciones para la nueva administración, seguidamente se analiza el estado de las relaciones brasileño-estadounidenses desde los cuatro ejes antes establecidos (relación económica, energía/medio ambiente, defensa y asuntos sociales), poniendo especial énfasis en el estado de las instituciones bilaterales.

Englobando todas las áreas anteriores, se coloca el U.S.-Brazil Strategic Partnership Dialogue, el cual fue formalizado el 13 de septiembre de 2019. Es conducido directamente por el ministro de Relaciones Exteriores de Brasil y el secretario de Estado de Estados Unidos y se posiciona, en la actualidad, como el principal instrumento para la coordinación de acciones conjuntas. Sin embargo, es importante señalar que esta no es una herramienta nueva, sino que tiene sus raíces en el U.S.-Brazil Economic Partnership Dialogue, el cual funcionó entre el 2007 y 2008, durante la administración Bush (U.S. Department of State, 2009), y que luego fue relanzado en el 2011 con el nombre de U.S.-Brazil Global Partnership Dialogue, en el transcurso de la administración Obama (U.S. Department of State, 2011). El ahora llamado Strategic Partnership Dialogue 
tiene tres pilares de acción que son apoyar a la gobernabilidad democrática, perseguir la prosperidad económica y fortalecer tanto la defensa como la seguridad (Office of the Spokesperson, 2019a, 2019b).

Al haber concluido la administración Trump, fue planteada la necesidad de una Brazil-U.S. Binational Institution con mayor capacidad de maniobra y recursos (Runde \& Kohan, 2021). Si bien el gobierno de Biden podría retomar dicha iniciativa, es previsible que en un primer momento el principal instrumento para el diálogo político con Brasil siga siendo el Strategic Partnership Dialogue.

\subsection{Relaciones económicas: el motor de la asociación bilateral}

En el 2009, Estados Unidos se convirtió en el segundo socio comercial de Brasil, al ser desplazado por China. En lo que ha sido la segunda mitad de la década de los 10, las exportaciones brasileñas hacia Estados Unidos se mantuvieron muy estables, pues pasaron de \$24 mil millones en el 2015 (año en que la presidenta Rousseff inició su segundo mandato) a \$29 mil millones en el 2019 (primer año del gobierno Bolsonaro). Sin embargo, mientras la balanza comercial con China ha sido mayoritariamente superavitaria para Brasil, a lo largo del siglo XXI (con una balanza favorable por \$28 mil millones en el 2019), con Estados Unidos ha ocurrido lo contrario, pues tal balanza se ha tornado deficitaria para el país sudamericano (registra un valor de $-\$ 557$ en el 2019). En ese sentido, el principal interés para Brasil es reequilibrar la balanza comercial, especialmente porque Estados Unidos ha mantenido cuotas tarifarias, prohibiciones fitosanitarias y barreras técnicas que obstaculizan la llegada de productos brasileños o reducen su competitividad, especialmente en el ámbito agrícola (Embaixada do Brasil em Washington, 2018).

Ahora bien, en cuanto a las instituciones bilaterales comerciales, la instancia más antigua es el Brazil-U.S. Business Council (BUSBC), el cual pertenece a la U.S. Chamber of Commerce, tiene su sede en Washington, D. C. y ha desempeñado un rol muy activo en el llamamiento a ambos gobiernos, para tomar medidas concretas orientadas hacia la institucionalización de las relaciones bilaterales. El BUSBC ya ha tenido comunicación directa con Joe Biden desde sus años como vicepresidente, pues fue uno de los firmantes de las Top policy recommendations, en la preparación de su primera visita a Brasil en el 2013, y ha sido coorganizador de la Brazil-U.S. Business Summit (la última fue realizada en el 2015). Uno de los momentos de mayor actividad del BUSBC fue durante la administración Obama, por lo cual, muy posiblemente, este 
adquirirá un nuevo peso como interlocutor en las relaciones bilaterales, durante la administración Biden.

Otro instrumento importante es el U.S.-Brazilian Commercial Dialogue, creado en el 2006 (durante las presidencias de George W. Bush y Lula da Silva), el cual tiene cinco grupos de trabajo: Asociación Comercial, Industria e Inversión, Servicios, Propiedad Intelectual y Estándares. La última reunión plenaria del Dialogue fue la edición 18 realizada el 14 de mayo de 2020 (modalidad virtual), en la cual se definieron como prioridades estratégicas: facilitar el movimiento de mercancías a través de las fronteras; promover buenas prácticas regulatorias; mejorar el entendimiento de las regulaciones técnicas estadounidenses y brasileñas; aumentar el acceso a las normas y metodologías de medición; incrementar el acercamiento a la protección de la propiedad intelectual; instar la economía digital (U.S.-Brazil Commercial Dialogue, 2020). Es muy previsible que la administración Biden dé continuidad e impulse aún más los trabajos realizados por el Commercial Dialogue, pues, sin importar las orientaciones partidistas en ambos países, este ha sido un mecanismo dinámico, fiable y eficaz para la cooperación bilateral.

Otro instrumento muy relevante es el U.S.-Brazil CEO Forum, el cual fue creado en Brasilia el 10-11 de octubre de 2007 (durante las presidencias de George W. Bush y Lula da Silva) y está copresidido tanto por el secretario de Comercio de Estados Unidos como por el ministro de Economía de Brasil. El Foro es de naturaleza público-privada, se reúne anualmente alternando sedes entre Brasil y Estados Unidos y cuenta con la participación de entre 10 y 12 integrantes del sector empresarial de cada país. En la 11. ${ }^{a}$ Reunión, realizada por videoconferencia, simultáneamente en Brasilia y Washington, en septiembre de 2020, los representantes del Foro remarcaron que "la facilitación del comercio sigue siendo una cuestión prioritaria para ambos países, dado el potencial de reducir las regulaciones innecesariamente gravosas, recortar los costos y aumentar los flujos comerciales entre Estados Unidos y Brasil" (U.S.Brazil CEO Forum, 2020).

Por último, una de las más grandes prioridades en las relaciones económicas entre ambas naciones estudiadas se encuentra en el Agreement on Trade and Economic Cooperation (ATEC), firmado el 18 de marzo de 2011 (Government of the United States of America and the Government of the Federative Republic of Brazil, 2011). Este acuerdo tuvo un avance significativo en el gobierno de Trump, ya que se logró una actualización importante el 19 de octubre de 2020, con la signatura del Protocol on Transparency and Trade Rules, que consta de tres anexos: Facilitación del Comercio y Administración Aduanera; Buenas Prácticas Regulatorias, y Lucha contra la Corrupción (Government of the 
United States of America and the Government of the Federative Republic of Brazil, 2020). Tal actualización es fundamental, pues mejora las oportunidades para el comercio, al reducir las barreras no-arancelarias, especialmente a través de la simplificación de procedimientos burocráticos.

Muy posiblemente, la administración Biden dará continuidad a estas acciones e instrumentos, apoyándose en los actores institucionalizados. Debido al interés de Brasil por un mejor acceso al mercado estadounidense, podría contemplarse la conclusión de un acuerdo integral de libre comercio, así como de un convenio bilateral de inversiones en el mediano-largo plazo (Neto, Godinho, Hyatt \& Schineller, 2020). Además, dada la importancia de los asuntos monetarios en la relación bilateral para el presidente Biden (ver gráfico 4), podría ser relanzado el U.S.-Brazilian Economic and Financial Dialogue, pues es una herramienta poco desarrollada, pero que tiene por objetivo promover posiciones comunes en asuntos de economía global.

\subsection{Energía y cambio climático: prioridades reemergentes}

El medio ambiente es ahora el área más sensible de la relación Brasil-Estados Unidos. Es importante destacar que Brasil es un actor fundamental para el combate del cambio climático, pues posee la selva tropical con la mayor biodiversidad del mundo y alberga el $60 \%$ de la Amazonia. Sin embargo, con el gobierno Bolsonaro, Brasil pasó de ser un líder global a una amenaza en el cambio climático (Viscidi \& Graham, 2019).

Ahora bien, al impulsar el tema medioambiental como uno de los asuntos prioritarios en su campaña a la presidencia, Biden propuso la creación de un fondo internacional de \$20 000 millones para preservar la Amazonia en Brasil, pero, al mismo tiempo, advirtió que "el Presidente Bolsonaro debería saber que si Brasil no es un custodio responsable de la selva amazónica, entonces mi administración reunirá al mundo para asegurar que el medio ambiente esté protegido" (Americas Quarterly, 2020). Esto generó una reacción desafiante del presidente brasileño, quien respondió por Twitter: "Brasil ya no acepta sobornos, demarcaciones criminales o amenazas infundadas. Nuestra soberanía no es negociable" (Bolsonaro, 2020).

Hay pocas dudas de que la apuesta política del presidente Bolsonaro era por la reelección de Trump. Por ello, con la victoria de Biden, Brasil parte de una posición muy desfavorable; el tema medioambiental resulta el principal punto de desencuentro y fricción con la nueva administración estadounidense. No obstante, aunque prevalezcan las diferencias ideológicas entre los líderes de 
ambos países, las acciones brasileño-estadounidenses en materia de combate al cambio climático serían reencausadas y relanzadas, por medio de las instituciones bilaterales, pues estas son amplias y sólidas.

Sus raíces se encuentran en la Joint Statement on the United States-Brazil Common Agenda for the Environment, firmada en 1995 durante las presidencias de Clinton y Cardoso, la cual generó una serie de reuniones bilaterales anuales que funcionaron hasta el 2003. Posteriormente, con la administración Bush, se signó la Joint statement announcing intention to expand and intensify bilateral efforts to address climate change en el $2004 \mathrm{y}$, con el presidente Obama, este tema fue reposicionado en la agenda bilateral, al acordar, junto con el presidente Da Silva, un Memorandum of Understanding on Cooperation Regarding Climate Change en el 2010. Dicho instrumento sirvió de base para que en el 2015 se lograra el U.S.-Brazil Joint Statement On Climate Change, con el cual las dos naciones se comprometieron a intensificar la colaboración frente a los desafíos del cambio climático y crearon para ello el High Level United StatesBrazil Climate Change Working Group (CCWG), que tuvo reuniones en el 2015 y 2016. En los siguientes años, la agenda de trabajo brasileño-estadounidense en materia de cambio climático entró en un impasse por el negacionismo de sus presidentes: Trump retiró a Estados Unidos del Acuerdo de París y Bolsonaro buscó seguir los mismos pasos, pero este último fue presionado por Francia, que condicionó la negociación del tratado de libre comercio Unión EuropeaMercosur a su permanencia en dicho acuerdo.

Con la llegada de Joe Biden a la presidencia, este es el tema que ha sufrido el mayor ajuste, pues la crisis climática ha sido colocada en el centro de la política exterior y la seguridad nacional de Estados Unidos. En ese sentido, no cabe duda de que la administración Biden presionará fuertemente al gobierno Bolsonaro, ya que, según la orden ejecutiva del 27 de enero de 2021, se han dado instrucciones a varias agencias del gobierno para desarrollar "un plan para promover la protección de la selva amazónica" (The White House, 2021). Aun cuando no sea posible el diálogo en el nivel presidencial, Brasil será sujeto de varias iniciativas generadas desde la Casa Blanca, por lo cual es previsible que el CCWG sea reactivado. Este mecanismo estaría llamado a ser la institución bilateral más importante para alcanzar nuevos entendimientos y organizar acciones conjuntas frente al cambio climático.

Considerando lo anterior, la agenda climática se encuentra estrechamente relacionada con la energía, puesto que, mientras no se reduzca el uso de los combustibles fósiles como principal recurso energético, será muy difícil alcanzar las metas del Acuerdo de París de la COP21. En esta línea, resulta esencial señalar que Estados Unidos es el segundo mayor consumidor de energía en el 
mundo, así como el segundo productor de energía renovable (después de China, en ambos casos); mientras que Brasil es el octavo consumidor de energía, pero también, el tercer productor de energía renovable en el nivel mundial (más de la mitad es hidroeléctrica). Estas son dos potencias energéticas que colaboran entre sí, dado que después de Canadá y México, la relación hemisférica más importante que Estados Unidos tiene en este campo es con Brasil. Por ello, la coordinación de acciones entre ambos gigantes energéticos es estratégica para los esfuerzos globales y, por dichas razones, la cooperación energética ha sido uno de los temas más desarrollados en los ligámenes bilaterales.

En el 2003, Bush y Da Silva acordaron lanzar la U.S.-Brazil Energy Partnership y, en 2007, discutieron la importancia tanto del etanol como del biodiesel en la soberanía energética. La cooperación bilateral continuó durante las presidencias de Obama y Rousseff, quienes en el 2011 crearon el Strategic Energy Dialogue, un mecanismo público-privado que se orientaba a promover la seguridad energética, contribuir al desarrollo económico entre ambos países y reducir las emisiones de carbono. Incluso, en los gobiernos de Trump y Bolsonaro, las partes involucradas establecieron, en el 2019, el United States-Brazil Energy Forum (USBEF), presidido por el secretario de Energía de Estados Unidos y el ministro de Minas y Energía de Brasil, con la tarea de fortalecer la cooperación energética, así como de facilitar el comercio y las inversiones en este sector. El USBEF celebró su primera reunión en febrero de 2020 y acordó un plan de acción conjunta para los meses siguientes. En este sentido, se puede esperar que en la administración Biden siga siendo intensa la cooperación energética entre Estados Unidos y Brasil, y que el USBEF se desempeñe como principal interlocutor, aunque con una agenda de trabajo mayormente influida por las prioridades del cambio climático.

\subsection{Cooperación militar: del estatus de Major non-NATO Ally (MNNA) al reajuste del tema militar en la agenda bilateral}

Estados Unidos y Brasil tienen una larga historia de cooperación en materia de defensa. Algunos de los momentos más significativos fueron en la II Guerra Mundial, cuando Brasil autorizó temporalmente a Estados Unidos el uso de algunas de sus bases militares y le proveyó de tropas, y, en 1952, cuando fue firmado el Acuerdo de Asistencia Militar Brasil-Estados Unidos, con el objetivo de garantizar la defensa en el hemisferio occidental. Dicho convenio fue denunciado en 1977 por el presidente militar Ernesto Geisel (Presidência da República, 1977), por acusaciones estadounidenses sobre la situación 
de los derechos humanos en Brasil (D’Araújo, s. f.). A partir de entonces, la cooperación militar se mantuvo relativamente estancada.

Fue hasta finalizada la Guerra Fría y el retorno de Brasil a la democracia, cuando ambos países hicieron mayores esfuerzos para incrementar la cooperación militar. De esta forma, en el 2010, firmaron el Defense Cooperation Agreement y el General Security of Military Information Agreement, con el objetivo de profundizar vínculos militares y explorar nuevas áreas de interés común. Con base en ello, Obama y Rousseff avalaron la creación del Defense Cooperation Dialogue (DCD) en el 2012 y del Defense Industry Dialogue (DID) en el 2015, mecanismos que simbolizaron el punto de mayor acercamiento militar, después del Acuerdo de Asistencia Militar de 1952. Con la llegada de Trump y Bolsonaro, ambas herramientas continuaron trabajando de manera muy dinámica.

Sin embargo, más que un impulso a la cooperación militar, lo que en realidad hicieron Trump y Bolsonaro fue sobredimensionar este campo. Como se mostró en el gráfico 2, los asuntos de seguridad y defensa fueron los primeros en importancia en las relaciones brasileño-estadounidenses, según los discursos de Trump y Bolsonaro, sin abandonar la vinculación de este tema, directamente, con la crisis en Venezuela. El sobredimensionamiento de lo militar fue culminado en julio de 2019, cuando Trump designó a Brasil el estatus de MNNA (The White House, 2019b), un nombramiento que otorga privilegios militares y económicos a los países involucrados, en reconocimiento a la estrecha relación con Estados Unidos?.

Con la llegada de Joe Biden, el asunto de la cooperación militar vuelve a sus cauces institucionales, es decir, Brasil mantendrá los privilegios que le brinda su nuevo estatus de MNNA, al tiempo que el DCD y el DID seguirán operando con normalidad, pero los asuntos de defensa quedarán relegados en la agenda bilateral al tercer lugar, priorizando antes los temas económico y energético/ ambiental antes expuestos.

7 Existen 17 países con el estatus de Major non-NATO Ally (MNNA). Los únicos 2 países latinoamericanos son Argentina (designado en 1998 con el gobierno de Carlos Menem) y ahora Brasil. Los demás son Afganistán, Australia, Bahréin, Egipto, Israel, Japón, Jordania, Corea, Kuwait, Marruecos, Nueva Zelanda, Pakistán, Filipinas, Tailandia y Túnez. 


\subsection{Asuntos sociales: relanzamiento de una cooperación de larga tradición}

En buena medida, la cooperación entre Brasil y Estados Unidos descrita anteriormente está sustentada en las interacciones sociales de larga data, existentes entre ambos países. Los principales rubros son educación y tecnología, pero también se puede mencionar la salud. Estos se encuentran consistentemente institucionalizados y es un interés de Estados Unidos que se profundicen, tanto en el nivel bilateral como multilateral, razón por la cual la administración Trump impulsó arduamente la incorporación de Brasil a la Organización para la Cooperación y el Desarrollo Económicos (OCDE).

En cuanto al ámbito educativo, existe una amplia red de programas, entre ellos, el Fulbright Program (establecido en 1957), que ha sido el más importante en la investigación y generación de conocimiento; el Programa de desarrollo de profesores de inglés (desde 2011), que ha ayudado en la profesionalización del personal docente de lengua inglesa; la iniciativa 100,000 Strong in the Americas, un programa de la Casa Blanca para financiar propuestas innovadoras de mejora laboral; EducationUSA, que promueve los intercambios universitarios; US-Brazil i-Corps, que se ha implementado para la educación emprendedora de startups, por mencionar los más destacados. Todas estas iniciativas se encuentran enmarcadas por el Agreement on Science and Technology between the United States and Brazil (firmado en 1984 y extendido en 1994) y por la US-Brazil Partnership for Education (firmada en 1997 y relanzada en el 2007). El convenio más importante en este ámbito alcanzado por Trump y Bolsonaro fue la firma del Technological Safeguards Agreement (AST), por el cual Brasil protege, contra copia o robo, las tecnologías estadounidenses y garantiza, con ello, las condiciones para el intercambio comercial.

Entre todo este compendio de agencias y acuerdos, la más importante es la Joint Commission Meeting on Science and Technology Cooperation (JCM), creada en el 2006. Es presidida por el ministro de Ciencia, Tecnología e Innovación de Brasil y el director del Instituto Nacional de Normas y Tecnología de Estados Unidos y cuenta con la participación de representantes de más de 30 agencias especializadas de ambos países. La JCM celebró su quinta reunión en marzo de 2020, por lo cual es asumible que seguirá siendo el mecanismo más destacado para la coordinación de labores científicas y educativas en el ámbito binacional.

Finalmente, ante el brote de coronavirus en el 2020, un tema que adquirió especial trascendencia en la cooperación internacional, aludiendo a lo global, fue la salud. Al respecto, Estados Unidos y Brasil tienen una relevante trayectoria de colaboración en investigación biomédica, prevención de enfermedades, 
análisis de datos e intercambio de información en materia de salud. Sin embargo, las posturas escépticas de Trump y Bolsonaro sobre la magnitud de la COVID-19, convirtió a sus países en los dos con mayor cantidad de muertes por la enfermedad en el 2020. Si bien Biden ha hecho importantes ajustes en la gestión de la pandemia, los esfuerzos del nuevo gobierno se encuentran, de momento, centrados en resolver el problema interno. De ahí que el 25 de enero de 2021, la administración Biden determinó restringir la entrada a Estados Unidos de no-ciudadanos provenientes de ciertos países considerados de alto riesgo, entre ellos Brasil.

\section{Conclusión}

Joseph Biden es posiblemente uno de los políticos estadounidenses con más experiencia en relaciones internacionales en Washington, primero por su trabajo como senador y presidente del Comité de Relaciones Exteriores del Senado y, luego, como vicepresidente, cuando se desempeñó como actor clave en la administración Obama, al realizar numerosos viajes que ampliaron y consolidaron la presencia de Estados Unidos en el mundo.

A raíz de la elección de Biden como el presidente 46 de los Estados Unidos, en noviembre de 2020, un número creciente de comentarios y análisis comenzaron a ser difundidos sobre el giro que podría tomar la política exterior de la Casa Blanca. Al respecto, es posible señalar que las relaciones de Estados Unidos con América Latina han entrado en un proceso de relanzamiento. Este enfoque se basa en su amplia destreza, pues el gobernante fue el primer funcionario de alto nivel de la administración Obama en viajar a Centro y Sudamérica (Costa Rica y Chile, respectivamente, en marzo de 2009), así como el último en visitar la región (Colombia, diciembre de 2016) antes del traspaso de poder a Trump. Además, con sus 19 visitas a países del área, desarrolló un conocimiento de primera mano sobre la diversa y compleja realidad de América Latina. Por todo ello, al inicio de la administración Biden, es factible proyectar cambios significativos en las relaciones de Washington con toda la región, pero con un trato diferenciado para cada nación.

Aunque la llegada de Biden no ha sido del agrado del presidente Bolsonaro, las circunstancias obligan a ambas partes a encontrar los mecanismos para el trabajo conjunto. En las relaciones brasileño-estadounidenses, las instituciones bilaterales están ampliamente desarrolladas. Estas han ayudado históricamente no solo a mantener el desarrollo de la agenda bilateral, sino que también permitirán, en el futuro, el diálogo entre dos gobiernos que se muestran ideológicamente distantes, así como un reajuste en la jerarquía de los temas: 
primero las relaciones económicas, luego lo energético y medioambiental, y, finalmente, lo militar y social.

Con esta investigación, se ha examinado no únicamente la situación de una de las relaciones más importantes de las Américas, sino también la relevancia de las instituciones bilaterales en la política exterior, frente a gobiernos de orientación populista.

\section{Referencias}

Americas Quarterly. (2020). Joe Biden Answers 10 Questions on Latin America, Americas Quarterly. https://www.americasquarterly.org/article/ updated-2020-candidates-answer-10-questions-on-latin-america/

Biden, J. (2013). Remarks by the Vice President on U.S.-Brazil Relations, Rio de Janeiro, Brazil. The White House - President Barack Obama. http://bit. ly/355ZyZj

Bodman, S., Wolfersohn, J. \& Sweig, J. (2011). Independent Task Force Report No. 66. New York: Council on Foreign Relations.

Bolsonaro, J. M. (2020). O que alguns ainda não entenderam é que o Brasil mudou... [Tweet]. Twitter: https://twitter.com/jairbolsonaro/ status/1311325732866588678

Briceño, J. (2016). Del Panamericanismo al ALCA: la difícil senda de las propuestas de una comunidad de intereses en el continente americano (I). Anuario Latinoamericano, 3, 145-167. Doi: 10.17951/al.2016.3.145

Briceño, J. (2017). Del panamericanismo al ALCA: la difícil senda de las propuestas de una comunidad de intereses en el continente americano (II). Anuario Latinoamericano, 4, 139-162. Doi: 10.17951/al.2017.4.139

Burns, B. (1966). The Unwritten Alliance. Rio Branco and Brazilian-American Relations. New York: Columbia University Press.

Caballero, S. \& Crescentino, D. (2020). From the quest for autonomy to the dual break: structural and agential changes in Brazil's foreign policy during the 21st century. Revista Brasileira de Política Internacional, 63(1), 1-20. Doi: 10.1590/0034-7329202000111

Cardoso, F. H., Nunes, A., Amorim, C., Lafer, C., Resek, F., Serra, J., . . . Kalout, H. (2020). La reconstrucción de la política exterior brasileña. https://bit.ly/3iSget9 
Chryssogelos, A. (2017). Populism in Foreign Policy. Oxford Research Encyclopedia of Politics. https://oxfordre.com/politics/view/10.1093/ acrefore/9780190228637.001.0001/acrefore-9780190228637-e-467

Committee on Ways and Means. (2020). Ways and Means Democrats Strongly Oppose Expanding Economic Relations with President Bolsonaro's Brazil. https://bit.ly/3td6wWY

Cooper, A. \& Antkiewicz, A. (2008). Emerging power in global governance. Ontario: Wilfrid Laurier University Press.

Correa, J. (2020). Panamericanismo versus latinoamericanismo: tensión geopolítica y civilizacional. Analecta Política, 10(19), 56-76. Doi: 10.18566/apolit.v10n19.a03

D’Araújo, M. C. (s. f.). Acordo Militar Brasil-Estados Unidos (1952). https://bit.ly/34yqiB6

Destradi, S. \& Plagemann, J. (2019). Populism and International Relations: (Un)predictability, personalisation, and the reinforcement of existing trends in world politics. Review of International Studies, 45(5), 711-730. Doi: $10.1017 / \mathrm{S} 0260210519000184$

Embaixada do Brasil em Washington. (2018). Desafios eoportunidades à exportação de produtos brasileiros aos Estados Unidos. https://bit.ly/3pwcSyt

Fonseca, P., Paes, L. \& Cunha, A. (2016). The concept of emerging power in international politics and economy. Revista de Economia Política, 36(1 (142)), 46-69. Doi: 10.1590/0101-31572016v36n01a04

Frenkel, A. (2018). El mundo según Bolsonaro. La nueva política exterior de Brasil. http://bit.ly/3ocrfY8

Government of the United States of America and the Government of the Federative Republic of Brazil. (2011). Agreement on Trade and Economic Cooperation between the Government of the United States of America and the Government of the Federative Republic of Brazil. The Withe House - President Barack Obama: https://bit.ly/2Yq0nZk

Government of the United States of America and the Government of the Federative Republic of Brazil. (2020). Protocol to the Agreement on Trade and Economic Cooperation between Government of the United States of America and the Government of the Federative Republic of Brazil relating to trade rules and transparency. Office of the United States Trade Representative: https://bit.ly/39xM2R1

Gratius, S. (2008). Las potencias emergentes: ¿estabilizadoras o desestabilizadoras? https://www.academia.edu/11951113/ Las_potencias_emergentes_Estabilizadoras_o_desestabilizadoras 
Hakim, P. (2019). Remaking US-Brazilian Relations: The Odyssey of Trump and Bolsonaro. The Dialogue: http://bit.ly/3ctjGJt

Hurrell, A. (2012). Récits d'émergence: la fin du tiers monde? Critique internationale, 3(56), 17-38.

Mongan, M. (2020). Descifrando la política externa bolsonarista. Foreign Affairs Latinoamérica. http://revistafal.com/ descifrando-la-politica-externa-bolsonarista/

Morales, D. (2020). The semiperipheral States in the 21st century: Measuring the structural position of regional powers and secondary regional states. International Studies, 57(1), 20-50. Doi: 10.1177/0020881719880769

Neto, A., Godinho, D., Hyatt, K. \& Schineller, L. (2020). Comércio e investimentos Brasil-EUA: Aprofundando as Relações Econômicas Bilaterais. Atlantic Council. https://bit.ly/39ympj2

Nolte, D. (octubre de 2006). Potencias regionales en la politica internacional: conceptos y enfoques de análisis. German Institute of Global and Area Studies. https://www.giga-hamburg.de/en/publications/11575856potencias-regionales-pol\%C3\%ADtica-internacional-conceptos-enfoquesan $\% \mathrm{C} 3 \%$ A 1 lisis/

Office of the Spokesperson. (2019a). U.S.-Brazil Strategic Partnership Dialogue. https://bit.ly/36xsASB

Office of the Spokesperson. (2019b). Secretary Michael R. Pompeo And Brazilian Foreign Minister Ernesto Araujo Remarks to the Press. https://bit.ly/3j3kmXr

Padilla, E. (1954). The Meaning of Pan-Americanism. Foreign Affairs, 270281. Presidência da República. (1953). Decreto $n^{\circ}$ 33.044. https://www. foreignaffairs.com/articles/americas/1954-01-01/meaning-pan-americanism

Presidência da República. (1977). Decreto $n^{\circ}$ 79.376. https://bit.ly/3vDKKN1

Ribeiro Hoffmann, A. (2020). Brazil Under Bolsonaro. Latin American Policy, 11(3), 335-338. Doi: 10.1111/lamp.12200

Rocha, A. \& Morales, D. (2018). El poder nacional-internacional de los Estados. Una propuesta trans-estructural. Geopolitica(s), 9(1), 137-169. Doi: 10.5209/ GEOP. 57778

Runde, D. \& Kohan, A. (2021). Toward a Brazil-U.S. Binational Institution. Center for Strategic \& International Studies. http://bit.ly/3oXnAgw

Saraiva, M. (2012). Procesos de integración de América del Sur y el papel de Brasil: los casos del Mercosur y la Unasur. Revista CIDOB d'Afers Internacionals, 97/98, 87-100. http://www.jstor.org/stable/41635272 
Saraiva, M. \& Silva, Á. (2019). Ideologia e pragmatismo na política externa de Jair Bolsonaro. Relações Internacionais (64), 117-137. Doi: $10.23906 /$ ri2019.64a08

Scholvin, S. (2010). Emerging non-OECD countries: global shifts in power and geopolitical regionalization. Hamburg: GIGA German Institute of Global and Area Studies.

Schweller, R. (2011). Emerging powers in an age of disorder. Global Governance, 17, 285-297.

Soares de Lima, M. R. \& Hirst, M. (2006). Brazil as an intermediate state and regional power: action, choice and responsibilities. International Affairs, 82(1), 21-40.

Soreanu, C. (2008). As relações bilaterais Brasil-Estados Unidos (1989-2008). Nueva Sociedad, 86-103. https://bit.ly/3t56BMz

The White House. (2019a). Remarks by President Trump and President Bolsonaro of the Federative Republic of Brazil in Joint Press Conference. The White House - National Archives: http://bit.ly/368Yswp

The White House. (2019b). Designation of the Federative Republic of Brazil as a Major Non-NATO Ally. http://bit.ly/3pME7VO

The White House. (2021). Executive Order on Tackling the Climate Crisis at Home and Abroad. The White House - Briefing Room. http://bit.ly/36wt0IK

U.S. Department of State. (2009). United States-Brazil Economic Partnership Dialogue. U.S. Department of State - Archive. http://bit.ly/3aorqtA

U.S. Department of State. (2011). U.S.-Brazil Global Partnership Dialogue. U.S. Department of State - Archive: http://bit.ly/3ti8PYL

U.S.-Brazil CEO Forum. (2020). Joint Communique from the U.S.-Brazil CEO Forum. U.S. Department of Commerce. http://bit.ly/2YrFPj1

U.S.-Brazil Commercial Dialogue. (2020). Joint Statement of the 18th Edition of the U.S.-Brazil Commercial Dialogue. International Trade Administration U.S. Department of Commerce. http://bit.ly/2L1bm8s

Villarreal, A., Meyes, P. \& Schwarzenberg, A. (2020). U.S.-Brazil Economic Relations. Congressional Research Service. https://bit.ly/3j4S12J

Viscidi, L. \& Graham, N. (2019). Brazil Was a Global Leader on Climate Change. Now It's a Threat. Foreign Policy. http://bit.ly/3ctyJmp

Wehner, L. \& Thies, C. (2020). The nexus of populism and foreign policy: The case of Latin America. International Relations, 1-21. Doi: 10.1177/0047117820944430

Zakol, A. (2019). 'Rise of the rest': As hype and reality. International Relations, 1-16. Doi: $10.1177 / 0047117819840793$ 
